\title{
Review
}

\section{CAM and Cell Fate Targeting: Molecular and Energetic Insights into Cell Growth and Differentiation}

\author{
Carlo Ventura
}

\author{
Laboratory of Molecular Biology and Stem Cell Engineering, National Institute of Biostructures and Biosystems and
} Institute of Cardiology, S. Orsola-Malpighi Hospital, University of Bologna, 40138 Bologna, Italy

\begin{abstract}
Evidence-based medicine is switching from the analysis of single diseases at a time toward an integrated assessment of a diseased person. Complementary and alternative medicine (CAM) offers multiple holistic approaches, including osteopathy, homeopathy, chiropractic, acupuncture, herbal and energy medicine and meditation, all potentially impacting on major human diseases. It is now becoming evident that acupuncture can modify the expression of different endorphin genes and the expression of genes encoding for crucial transcription factors in cellular homeostasis. Extremely low frequency magnetic fields have been found to prime the commitment to a myocardial lineage in mouse embryonic stem cells, suggesting that magnetic energy may direct stem cell differentiation into specific cellular phenotypes without the aid of gene transfer technologies. This finding may pave the way to novel approaches in tissue engineering and regeneration. Different ginseng extracts have been shown to modulate growth and differentiation in pluripotent cells and to exert wound-healing and antitumor effects through opposing activities on the vascular system, prompting the hypothesis that ancient compounds may be the target for new logics in cell therapy. These observations and the subtle entanglement among different CAM systems suggest that CAM modalities may deeply affect both the signaling and transcriptional level of cellular homeostasis. Such a perception holds promises for a new era in CAM, prompting reproducible documentation of biological responses to CAM-related strategies and compounds. To this end, functional genomics and proteomics and the comprehension of the cell signaling networks may substantially contribute to the development of a molecular evidence-based CAM.
\end{abstract}

Keywords: acupuncture - differentiation - energy medicine - gene expression - stem cells

\section{Introduction}

Complementary and alternative medicine (CAM) is emerging as a heterogeneous subject encompassing wide-ranging holistic approaches to a diseased human being that include herbal medications, aromatherapy, acupuncture, yoga, tai chi and magnetic energy (1-4). Despite modern technology and chemistry, a number of chronic, degenerative and malignant diseases remain incurable by current western medicine. Coupled to this fact is an awareness that even the most recent

For reprints and all correspondence: Carlo Ventura, Laboratory of Molecular Biology and Stem Cell Engineering, National Institute of Biostructures and Biosystems and S. Orsola-Malpighi Hospital, Institute of Cardiology, Pavilion 21, Via Massarenti N. 9, University of Bologna, Bologna 40138, Italy. Tel: +39-051-6363605, 340339; Fax: +39-051-344859;

E-mail: cvent@libero.it, carlo.ventura@unibo.it therapeutic armamentarium provided by western medicine is still remarkably crude compared with the finely tuned complexity of biological systems. It is now becoming increasingly evident that such complexity is fashioned at multiple interconnected levels and is controlled by an interplay between cell signaling, gene expression, nucleosomal assembly, the establishment of multifaceted transcriptional motifs and the temporal and spatial organization of chromatin in loops and domains. Moreover, recently developed nanoscale science and technology interventions are providing a remarkable glimpse into how living organisms may organize nanoarchitectonics at a hierarchical level of assembly to produce specific cellular phenotypes and functions. Within this context, ancient practices have been revisited as CAM tools potentially impacting on major human diseases, and old compounds from traditional Chinese medicine have been shown to have features

(C) The Author (2005). Published by Oxford University Press. All rights reserved.

The online version of this article has been published under an open access model. Users are entitled to use, reproduce, disseminate, or display the open access version of this article for non-commercial purposes provided that: the original authorship is properly and fully attributed; the Journal and Oxford University Press are attributed as the original place of publication with the correct citation details given; if an article is subsequently reproduced or disseminated not in its entirety but only in part or as a derivative work this must be clearly indicated. For commercial re-use, please contact journals.permissions@oupjournals.org 
characteristic of morphogenetic agents suitable for cell therapy and tissue repair.

These findings hold promises for a new era in CAM and raise the need for reproducible documentation of biological responses to CAM-related strategies and compounds (5). Such a dissection may require novel holistic experimental approaches in molecular biology and medicine involving an interconnected analysis of functional genomics and proteomics and an integrated comprehension of the underlying signal-transduction mechanisms.

The development of suitable in vitro and in vivo experimental models is now perceived as a crucial requirement in CAM. Nevertheless, a consistent number of CAM modalities are still awaiting more functional and molecular approaches that may further underpin CAM systems.

So far, a detailed analysis of cell signaling networks and gene expression patterning has been carried out for a limited number of CAM strategies, including acupuncture, the use of magnetic energy and targeted compounds from herbal medicine. This review is focused on the discussion of intriguing findings achieved in these fields with the aid of molecular and cellular biology approaches, showing that CAM may be much more profound than conventional science has hitherto been prepared to admit.

\section{Acupuncture and Gene Expression}

Growing evidence indicates a close relationship between the application of acupuncture and the modulation of endorphinergic systems. One of the most surprising and fascinating insights in recent analysis of the interplay among these systems and acupuncture is the finding that this treatment is able to elicit remarkable changes in the expression of specific endorphin genes. Experimental studies performed in rats revealed that 2 and $100 \mathrm{~Hz}$ acupuncture primed consistent changes in brain expression of the three opioid genes proopiomelanocortin, preproenkephalin and prodynorphin (6). The spatial and temporal patterns of these changes varied in a frequency-dependent fashion. In particular, $2 \mathrm{~Hz}$ electroacupuncture mainly increased preproenkephalin gene expression without affecting the expression of the prodynorphin gene, which was in turn selectively increased following a $100 \mathrm{~Hz}$ treatment (6). In different experimental animal models, electroacupuncture was also found to increase brain expression of the $c$-fos and $c$-jun genes, as well as the expression of their related protein products (7-9). This is particularly rewarding since Fos is an important transcription factor selectively recognizing opioid gene promoters. Moreover, Jun and Fos are known to establish productive interactions leading to the formation of heterodimers, acting as strong transactivating complexes for genes playing a crucial role in cellular homeostasis. Within this context, concerted activation of Fos/Jun expression by electroacupuncture emerged as a molecular mechanism underlying the selective increase in prodynorphin gene expression as compared with the expression of the preproenkephalin gene (10). These effects were blocked by antisense oligonucleotides directed against $c$-fos and $c$-jun (10), suggesting that acupuncture may regulate gene expression by selectively affecting transcription factor assembly into specific multifunctional transcriptional complexes.

With respect to the cardiovascular system, application of $5 \mathrm{~Hz}$ electroacupunture at the level of the median nerves in rats proved effective in reducing myocardial ischemia and contractile dysfunctions provoked by partial experimental occlusion of the left anterior descending coronary artery or following ligation of its diagonal branch (11). In this experimental model, bradykinin was applied to the gallbladder to further impair myocardial dynamics, eliciting a number of reflex responses, including increases in left ventricular $\mathrm{dP} / \mathrm{dt}$, coronary blood velocity and myocardial oxygen demand (11). Under these experimental conditions, naloxone, a selective opioid receptor antagonist, was found to counteract the protective effects induced by electroacupuncture (12). These results indicate that even the cardiovascular responses elicited by acupuncture may be mediated, at least in part, by the activation of endorphinergic mechanisms.

These observations and the ability of acupuncture to activate endorphin gene expression and signaling may have further biomedical implications. It is now clear that endorphins play an important role in ischemic preconditioning, a mechanism through which myocardial tissue exposed to repeated minor ischemic events acquires substantial protection against a major ischemic insult. Such a view is supported by a number of interrelated observations. First, in freshly isolated adult rabbit cardiomyocytes subjected to simulated ischemia, cell death was remarkably prevented by dynorphin B, an endogenous kappa opioid receptor agonist, and by the synthetic ligand of delta receptors Met5-enkephalin. Such an effect was specific in nature and involved sarcolemmal and mitochondrial ATPsensitive $\mathrm{K}^{+}$channels (13). Second, the cardioprotective benefits of ischemic preconditioning can be transferred from preconditioned to virgin acceptor hearts via coronary effluent transfusion, implying the presence of hormonal preconditioning factor(s) (14). In this study, material released into the coronary effluent of isolated buffer-perfused rabbit hearts during preconditioned ischemia/reperfusion or normoxic perfusion was concentrated by reverse phase chromatography. Treatment with preconditioned concentrate but not with normoxic effluent evoked cardioprotection equivalent to that afforded by conventional preconditioning (14). Naloxone nullified the protection achieved by transfer of preconditioned concentrate. These results indicate that coronary effluent from preconditioned hearts evokes a cardioprotective effect via a mechanism requiring an intact opioid receptor system (14). Third, in isolated perfused rat hearts the stimulation of kappa opioid receptors with the synthetic agonist U-50488H not only reduced the size of the infarct induced by ischemia, mimicking the protective effect of ischemic preconditioning, but elicited a significant amelioration of ischemia-induced arrhythmias (15).

In this light, acupuncture may be viewed as a therapeutic system considerably more complex than expected since its effects on crucial organs and apparatuses may involve a deep 


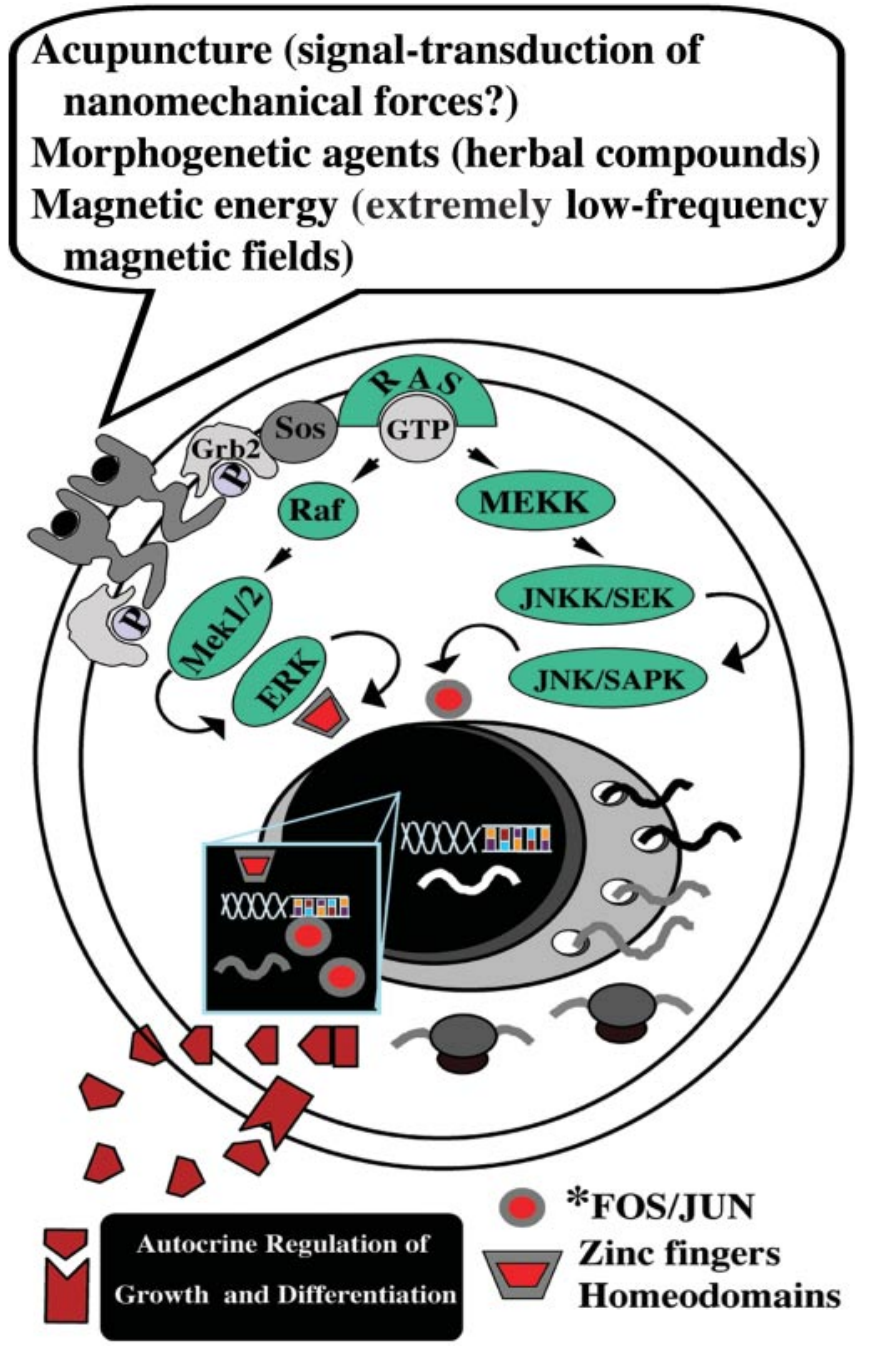

Figure 1. Putative signaling networks encompassed by CAM strategies. Increasing evidence indicates that acupuncture leads to the expression of $c$-fos and c-jun genes and their related protein products, which act as growth regulatory transcription factors. Concerted activation of Fos/Jun expression by electroacupuncture has emerged as a molecular mechanism underlying the increase in the expression of targeted endorphin genes. Additionally, old nutraceuticals commonly used in Chinese traditional medicine have been found to have the potential for differentiating logics, acting on cell renewal and lineage commitment in pluripotent cells. Exposure to magnetic energy may ultimately result in the activation of signaling network(s) previously believed to be activated solely in the presence of a growth factor. Future directions may involve the analysis of CAM-related approaches within the context of the molecular examination of a number of signal-transduction mechanisms, including the activation of selected G-protein coupled receptors and the interplay between the subcellular redistribution patterning of defined kinase isozymes and the activation of tissue-restricted transcription factors (a representative scheme of a tyrosine receptor kinase signaling is shown). The analysis of long-lived signals, involving the establishment of autocrine feedforward circuits, should also be envisioned.

impact on the transcriptional and signaling machinery at the cellular level (Fig. 1). A large body of experimental studies in suitable in vivo and in vitro models should be envisioned to assess the molecular basis of acupuncture-evoked responses.

\section{Magnetic Fields and Stem Cell Differentiation: A Novel Frontier in Phenotyping Design}

Magnetic fields (MFs) have been shown to affect proliferation and growth factor expression in cultured cells (16-18) and to interfere with endorphinergic and cholinergic systems in intact organisms (19-21). MFs have been found to induce increased levels of hsp70, affording cytoprotection in different cell types (22), and have been reported to promote bone formation and to repair augmenting fracture and post-surgery healing $(23,24)$. Nevertheless, the possibility that the activation of a coordinated program of cell differentiation or the specification of a cellular architectural plan may represent the molecular underpinning of the MF effect has long awaited compelling experimental evidence. We have previously shown that exposure to extremely low frequency MFs increased the expression of the prodynorphin gene, encoding for the dynorphin family of opioid peptides, in adult rat ventricular cardiomyocytes (25). This effect was associated with the synthesis and secretion of dynorphin B (25), a bioactive gene product selectively acting on kappa opioid receptors coupled to phosphoinositide turnover, and regulation of cytosolic calcium homeostasis and contractility in the myocardial cell (26-28). It is now clear that endorphins may behave as growth factors remarkably affecting cell growth and differentiation. To this end, we have also provided evidence that dynorphin $\mathrm{B}$ primed cardiac differentiation in embryonal carcinoma cells (29) and that a dynorphinergic system acted as a major conductor of cardiogenesis in multipotent mouse embryonic stem (ES) cells $(30,31)$. Based on these findings we have recently investigated whether MF may be able to trigger endorphin gene expression even in ES cells and whether, if so, MF may commit these cells to the specification of a targeted lineage. For this purpose, mouse ES cells were exposed to a sinusoidal MF $(50 \mathrm{~Hz}$, $0.8 \mathrm{mT} \mathrm{rms}$ ) generated by a system consisting of two identical apparatuses each bearing four coaxial circular coils placed horizontally in a commercial $\mathrm{CO}_{2}$ incubator, and thus parallel with respect to the surface of the culture well plates. The geometry of the coil system was numerically calculated in order to optimize the extension of the MF uniformity. Each coil was wound by a pair of parallel wires so that, according to different connections, the current could flow either in the same direction ('wound configuration'), generating the active exposure, or in the opposite direction ('counter-wound configuration'), allowing a sham exposure. During the experiments, performed blind, the temperature was monitored by means of a thermoresistor. The local geomagnetic $(45 \mu \mathrm{T})$ field was measured using a gaussmeter (F.W.Bell 7010) and the AC background MF $(0.05 \mu \mathrm{T})$ by means of a sensitive probe (EMDEXII, Enertech).

Under these experimental conditions, ES cell exposure to MF was found to increase remarkably the expression of genes encoding for tissue-restricted transcription factors (Fig. 2), determining a consistent upregulation of GATA-4 and Nkx2.5 mRNA (32). These genes encode respectively for a zinc finger containing transcription factor and a homeodomain 


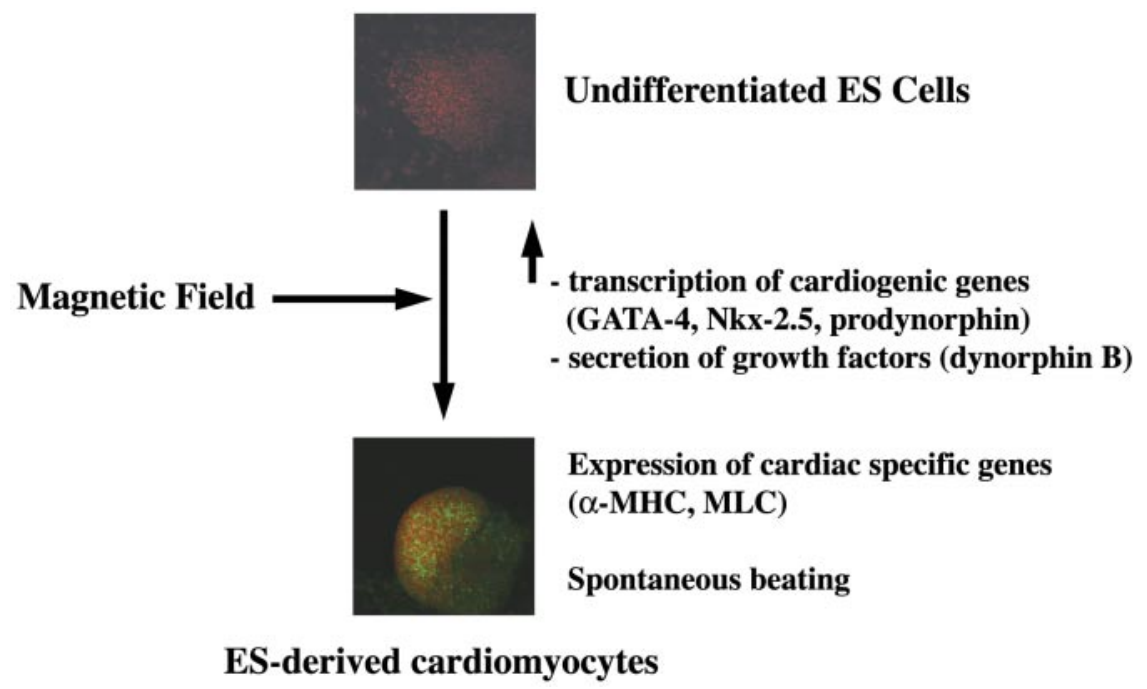

Figure 2. Stem cell exposure to magnetic energy leads to targeted lineage specification. As shown in the diagram, exposure of undifferentiated mouse embryonic stem cells (GTR1 cell line) to extremely low frequency magnetic fields primes the transcription of cardiac lineage promoting genes and the synthesis and secretion of dynorphin B, acting as an orchestrator of signaling and gene expression patterning required for the appearance of a myocardial phenotype (for details see the section 'Magnetic fields and stem cell differentiation: a novel frontier in phenotyping design'). Cardiomyocytes (lower panel) express cardiac-specific markers, including $\alpha$-myosin heavy chain ( $\alpha$-MHC) and myosin light chain-2V (MLC). ( $\alpha$-MHC is stained green with the MF 20 mouse antimyosin monoclonal antibody; DNA is visualized with prospidium iodide. Bio-Rad Microradians confocal microscope, $\times 20$ objective.)

that have been shown to be essential for cardiogenesis in different animal species $(33,34)$, including humans (35). MF also enhanced prodynorphin mRNA expression and the levels of dynorphin B in both embryoid bodies (EBs) and ES-derived cardiomyocytes and in their incubation media (32). This finding is particularly rewarding since we have shown that the prodynorphin gene and dynorphin B primed GATA-4 and Nkx-2.5 transcription $(29,30)$ and triggered protein kinase C (PKC) signaling through complex subcellular redistribution patterning of targeted PKC isozymes, another major requirement for ES cell commitment to the cardiac lineage (30). Additionally, dynorphin B was found to act as an agonist of nuclear opioid receptors coupling nuclear PKC activation to the transcription of cardiogenic genes, indicating that intracrine signals for cardiac differentiation may also be fashioned by the prodynorphin gene and its related peptides (31). Interestingly, nuclear run-off analyses performed in isolated ES nuclei indicated that the MF action occurred at the transcriptional level (32). The activation of a program of cardiogenic gene transcription was also associated with an increase in the expression of the cardiac-specific transcripts $\alpha$-myosin heavy chain and myosin light chain-2V (Fig. 2). On the whole, these transcriptional responses elicited a significant increase in the number of spontaneously beating cardiomyocytes, indicating that exposure to an MF resulted in a major enhancement of the cardiogenic potential of pluripotent stem cells (32). Noteworthy in this study is the fact that the expression of MyoD, a gene involved in skeletal myogenesis, was not affected in either EBs or ES-derived cardiomyocytes. On the contrary, the expression of neurogenin1, a neuronal specification gene, was slightly enhanced only in EBs. These observations exclude a generalized activation of repressed genes and suggest that coupling of MF with GATA-4, Nkx-2.5 and prodynorphin gene expression may represent a mechanism pertaining to ES cell cardiogenesis.

It has recently been proposed that stem cells are a novel tool for cellular therapy of damaged tissues. Unfortunately, their rescuing potential is limited by the fact that differentiating cells withdraw early from the cell cycle. Hence, the development of strategies affording high throughput of targeted lineages from pluripotent cells would have obvious biomedical implications. However, overexpression of tissue-specific genes by vector-mediated gene transfer to gain a high throughput of targeted lineages from pluripotent cells is a cumbersome approach that may perturb normal homeostasis in both stem cells and recipient tissues, and it is not readily envisionable in humans.

The observation that cell fate may be orchestrated by MF opens the new perspective of using magnetic energy to direct the differentiation processes of stem cells into a specific cellular phenotype without the aid of gene transfer technologies. These findings also prompt future investigations to shed additional light on the molecular events underlying the differentiating response primed by MF in ES cells and to assess whether such a response may be dependent on the field characteristics, including MF intensity, frequency and wave shape (signaling networks hypothetically encompassed by MF-related dynamics are depicted in Fig. 1).

\section{Ginseng and Cell Fate Patterning: Old Molecules and New Logics}

Ginseng is a commonly used nutraceutical. Mass spectrometric compositional analysis of American, Chinese, Korean and Sanqi ginseng revealed distinct 'sterol ginsenoside' fingerprints, especially in the ratio between a triol, $\mathrm{Rg} 1$, and a diol, 
$\mathrm{Rb} 1$, the two most prevalent constituents (36). The ginsenoside $\mathrm{Rg} 1$ was shown to promote proliferation of hippocampal progenitor cells (37). Ginsenosides Rh1 and Rh2 were found to induce differentiation of embryonal carcinoma cells in endoderm-like cells (38). This effect was prevented by a glucocorticoid receptor (GR) antagonist, and protein factors capable of specifically binding to the glucocorticoid responsive element (GRE) were detected in nuclear extracts of the ginsenoside-treated cells. Moreover, overexpression of GR by co-transfection of a GR expression vector and a GREluciferase vector enhanced the transactivation activity of the GRE promoter in the presence of the two different ginsenosides (38). The existing literature reports both wound-healing and antitumor effects of ginseng extracts through opposing activities on the vascular system. Using a Matrigel implant model and reconstituting the extracts with the aid of distinct ratios of the two ginsenosides $\mathrm{Rg} 1$ and $\mathrm{Rg} 2$, the dominance of $\operatorname{Rg} 1$ has been recently found to promote angiogenesis, whereas Rb1 exerted an opposing effect (36). Rg1 also elicited functional neovascularization into a polymer scaffold in vivo and the proliferation, chemoinvasion and tubulogenesis of endothelial cells in vitro (36). In contrast, Rb1 inhibited the earliest step in angiogenesis, the chemoinvasion of endothelial cells. The pro-angiogenic effects of $\operatorname{Rg} 1$ involved the expression of nitric oxide synthase (NOS) and the phosphatidylinositol-3 kinase-Akt pathway (36). Intriguingly, besides its crucial role in neovascularization, endothelial NOS is expressed by bone marrow stromal cells and has been found to be essential in the recruitment of stem and progenitor cells (39). It has been proposed that this contributes to impaired regeneration processes in ischemic heart disease patients, who are characterized by a reduced systemic NO bioactivity. In this regard, both inducible and endothelial NOS isoforms are prominently expressed during early stages of cardiomyogenesis and NO generation is required for cardiomyogenesis, since NOS inhibitors prevent the maturation of terminally differentiated cardiomyocytes in in vitro ES cell systems (40). The ability of ginsenosides to activate Akt signaling is also worthy of consideration. Within this context, transplantation of rat mesenchymal stem cells genetically engineered to overexpress Akt into the ischemic rat myocardium inhibited the process of cardiac remodeling by reducing intramyocardial inflammation, collagen deposition and cardiac myocyte hypertrophy (41). Akt-overexpressing cells regenerated $80-90 \%$ of lost myocardial volume and completely normalized systolic and diastolic cardiac function (41). Thus, mesenchymal stem cells genetically enhanced with Akt can repair infarcted myocardium, prevent remodeling and nearly normalize cardiac performance.

Transplantation of adult bone marrow-derived mesenchymal stem cells has been proposed as a strategy for cardiac repair following myocardial damage (42). However, poor cell viability associated with transplantation has limited the reparative capacity of these cells in vivo. In this light, chemical and signaling features from ginsenosides prompt the hypothesis that ginseng may contain potential differentiating molecules that have a role in cardiovascular rescue. Further studies involving molecular and cellular biology approaches and compelling in vivo validation are needed to address these issues. If the results are positive, an ancient compound may be the target for new logics in cell therapy and tissue engineering. (Fig. 1 traces working hypotheses on signaling and transcriptional patterning that may be investigated to uncover the molecular underpinning for the effects elicited by ginsenosides and other potential morphogenetic agents isolated from herbal compounds.)

\section{Wide-Ranging Transcriptional Profiling of CAM Responses: From Holistic Medicine to Holistic Molecular Biology}

The analysis of the experimental findings discussed in this review indicates that many CAM modalities may be impacting the transcriptional level of cell functioning and may be further exploited by genomic means. However, conventional techniques, including RT-PCR, northern blotting and RNase protection, allow the analysis of the expression of only a few genes at a time. Moreover, these techniques imply a selection 'a priori' of putative candidate genes intervening in the investigated responses, providing only a partial picture with no direct information about the abundance and timely patterning of transcriptional events.

The recent development of novel DNA nanotechnologies such as the serial analysis of gene expression (SAGE) and DNA microarrays has allowed a rapid and detailed analysis of thousands of transcripts, providing a revolutionary approach to the investigation of gene expression. SAGE is a nanoscale DNA biotechnology essentially based on two principles (43). First, a short nucleotide sequence tag of 9 bp contains sufficient information to uniquely identify a transcript, provided it is isolated from a defined position within the transcript. Second, concatenation of short sequence tags to form a 'concatemer' allows the efficient analysis of transcripts in a serial manner by the sequencing of multiple tags within a single clone. The sequence of the clones will allow the development of a profile for the gene expression. Quantitative data regarding the prevalence of an expressed gene will be derived from the frequency with which a given tag is found. To this end, the SAGE approach has led to the awareness of multiple gene expression patterning within a given experimental time course. Furthermore, new transcripts corresponding to novel tags have been identified.

Another experimental approach that can be used for multiple gene expression profiling is the DNA chip (44). In this method, oligonucleotides corresponding to candidate genes are deposited onto a glass surface to construct a DNA microarray. Fluorescent cDNAs are prepared from the different mRNA sources with the use of reverse transcriptase in the presence of fluoresceine- and lissamine-labeled nucleotide analogs. One set of cDNA is from the control sample and the other from the sample in which differential gene expression is being 
examined. The two fluorescent cDNAs are then allowed to hybridize to the DNA microarray and a pattern of fluorescence is detected by separately scanning the fluorescence emitted from excited fluoresceine and lissamine. Fluorescence analysis is ultimately used to monitor simultaneously both the overexpression and the downregulation of multiple genes within each sample investigated.

The use of these techniques in the analysis of large-scale transcriptional profiling is still at an early stage. However, it is conceivable that in the next few years these novel strategies may help to understand how the intricate machinery of gene expression is driven by the interplay of different signaltransduction pathways.

Evidence-based medicine is progressively switching from a strategy involving the assessment of single diseases at a time to a holistic approach centered on a diseased person, thereby recognizing a fundamental strategy that has long been established and validated in CAM. The identity of gene expression profiles potentially involved in a large number of CAM-related responses remains mostly enigmatic. The potential application of SAGE and DNA microarrays to CAM systems may conceivably lead to the identification and characterization of genes and signaling pathways that are involved in CAM modalities, thrusting forward further exploration of the role of selected identified genes in suitable animal models.

CAM disciplines, owing to their holistic nature, have long been thought to be far from influencing DNA dynamics and the molecular plight(s) of cell functioning. The findings discussed in the present review may therefore appear quite revolutionary for CAM and suggest that the CAM literature may shortly encompass a number of discoveries attained by the aid of conventional science techniques.

These perceptions and the future directions suggested by molecular genomics may offer a crucial contribution to the development of a molecular evidence-based CAM.

\section{References}

1. Cooper EL. Drug discovery, CAM and natural products. eCAM 2004;1: 215-17.

2. Müller WEG, Schröder HC, Wiens M, Perovic-Ottstadt S, Batel R, Müller IM. Traditional and modern biomedical prospecting: Part II-the benefits. eCAM 2004;1:133-44.

3. Satoh H. Comparative electropharmacological actions of some constituents from Ginkgo biloba extract in guinea-pig ventricular cardiomyocytes. eCAM 2004;1:277-84.

4. Irwin M, Pike J, Oxman M. Shingles immunity and health functioning in the elderly: Tai Chi Chih as a behavioral treatment. eCAM 2004;1:223-32.

5. Cooper EL. Complementary and alternative medicine, when rigorous, can be science. eCAM 2004;1:1-4.

6. Guo HF, Tian J, Wang X, Fang Y, Hou Y, Han J. Brain substrates activated by electroacupuncture of different frequencies (I): comparative study on the expression of oncogene c-fos and genes coding for three opioid peptides. Brain Res Mol Brain Res 1996;43:157-66.

7. Lee JH, Beitz AJ. Electroacupuncture modifies the expression of c-fos in the spinal cord induced by noxious stimulation. Brain Res 1992;577: $80-91$.

8. Lee JH, Beitz AJ. The distribution of brain-stem and spinal cord nuclei associated with different frequencies of electroacupuncture analgesia. Pain 1993;52:11-28.

9. Pan B, Castro-Lopes JM, Coimbra A. Chemical sensory deafferentation abolishes hypothalamic pituitary activation induced by noxious stimulation or electroacupuncture but only decreases that caused by immobilization stress. A c-fos study. Neuroscience 1997;4:1059-68.

10. Guo HF, Tian J, Wang X, Fang Y, Hou Y, Han J. Brain substrates activated by electroacupuncture (EA) of different frequencies (II): role of Fos/Jun proteins in EA-induced transcription of preproenkephalin and preprodynorphin genes. Brain Res Mol Brain Res 1996;43: 167-73.

11. Li P, Pitsillides KF, Rendig SV, Pan HL, Longhurst JC. Reversal of reflexinduced myocardial ischemia by median nerve stimulation: a feline model of electroacupuncture. Circulation 1998;97:1186-94.

12. Chao DM, Shen LL, Tjen-A-Looi S, Pitsillides KF, Li P, Longhurst JC. Naloxone reverses inhibitory effect of electroacupuncture on sympathetic cardiovascular reflex responses. Am J Physiol 1999;276:H2127-34.

13. Cao Z, Liu L, Van Winkle DM. Activation of delta- and kappa-opioid receptors by opioid peptides protects cardiomyocytes via KATP channels. Am J Physiol Heart Circ Physiol 2003;285:H1032-9.

14. Dickson EW, Blehar DJ, Carraway RE, Heard SO, Steinberg G, Przyklenk K. Naloxone blocks transferred preconditioning in isolated rabbit hearts. J Mol Cell Cardiol 2001;33:1751-6.

15. Wang GY, Wu S, Pei JM, Yu XC, Wong TM. Kappa- but not delta-opioid receptors mediate effects of ischemic preconditioning on both infarct and arrhythmia in rats. Am J Physiol Heart Circ Physiol 2001;280: H384-91.

16. Cossarizza A, Monti D, Bersani F, Cantini M, Cadossi R, Sacchi A, et al. Extremely low frequency pulsed electromagnetic fields increase cell proliferation in lymphocytes from young and aged subjects. Biochem Biophys Res Commun 1989;160:692-8.

17. Cadossi R, Bersani F, Cossarizza A, Zucchini P, Emilia G, Torelli G, et al. Lymphocytes and low-frequency electromagnetic fields. FASEB J 1992;6: 2667-74.

18. Cossarizza A, Angioni S, Petraglia F, Genazzani AR, Monti D, Capri M, et al. Exposure to low frequency pulsed electromagnetic fields increases interleukin-1 and interleukin-6 production by human peripheral blood mononuclear cells. Exp Cell Res 1993;204:385-7.

19. Thomas AW, Kavaliers M, Prato FS, Ossenkopp KP. Pulsed magnetic field induced 'analgesia' in the land snail, Cepaea nemoralis, and the effects of mu, delta, and kappa opioid receptor agonists/antagonists. Peptides 1997;18:703-9.

20. Vorobyov VV, Sosunov EA, Kukushkin NI, Lednev VV. Weak combined magnetic field affects basic and morphine-induced rat's EEG. Brain Res 1998:781:182-7.

21. Lai H, Carino MA, Horita A, Guy AW. Effects of a $60 \mathrm{~Hz}$ magnetic field on central cholinergic systems of the rat. Bioelectromagnetics 1993; 14:5-15.

22. Carmody S, Wu XL, Lin H, Blank M, Skopicki H, Goodman R. Cytoprotection by electromagnetic field-induced hsp70: a model for clinical application. J Cell Biochem 2000;79:453-9.

23. Ko J, Na DS, Lee YH, Shin SY, Kim JH, Hwang BG, et al. cDNA microarray analysis of the differential gene expression in the neuropathic pain and electroacupuncture treatment models. J Biochem Mol Biol 2002;35: $420-7$.

24. Ibiwoye MO, Powell KA, Grabiner MD, Patterson TE, Sakai Y, Zborowski $\mathrm{M}$, et al. Bone mass is preserved in a critical-sized osteotomy by low energy pulsed electromagnetic fields as quantitated by in vivo micro-computed tomography. J Orthop Res 2004;22: 1086-93.

25. Ventura C, Maioli M, Pintus G, Gottardi G, Bersani F. Elf-pulsed magnetic fields modulate opioid peptide gene expression in myocardial cells. Cardiovasc Res 2000;45:1054-64.

26. Ventura C, Guarnieri C, Stefanelli C, Cirielli C, Lakatta EG, Capogrossi MC. Comparison between alpha-adrenergic- and k-opioidergic-mediated inositol $(1,4,5)-\mathrm{P}_{3} /$ inositol $(1,3,4,5) \mathrm{P}_{4}$ formation in adult cultured rat ventricular cardiomyocytes. Biochem Biophys Res Commun 1991;179:972-8.

27. Ventura C, Capogrossi MC, Spurgeon HA, Lakatta EG. k-Opioid receptor stimulation increases $\mathrm{pH}$ and myofilament $\mathrm{Ca}^{2+}$ sensitivity in cardiac myocytes. Am J Physiol 1991;261:H1671-4.

28. Ventura C, Spurgeon HA, Lakatta EG, Guarnieri C, Capogrossi MC. Kappa and delta opioid receptor stimulation affects cardiac myocyte function and $\mathrm{Ca}^{2+}$ release from an intracellular pool in myocytes and neurons. Circ Res 1992;70:66-81.

29. Ventura C, Maioli M. Opioid peptide gene expression primes cardiogenesis in embryonal pluripotent stem cells. Circ Res 2000;87: 189-94. 
30. Ventura C, Zinellu E, Maninchedda E, Fadda M, Maioli M. Protein kinase $\mathrm{C}$ signaling transduces endorphin-primed cardiogenesis in GTR1 embryonic stem cells. Circ Res 2003;92:617-22.

31. Ventura C, Zinellu E, Maninchedda E, Maioli M. Dynorphin B is an agonist of nuclear opioid receptors coupling nuclear protein kinase $\mathrm{C}$ activation to the transcription of cardiogenic genes in GTR1 embryonic stem cells. Circ Res 2003;92:623-9.

32. Ventura C, Maioli M, Asara Y, Santoni D, Mesirca P, Remondini D, et al. Turning on stem cell cardiogenesis with extremely low frequency magnetic fields. FASEB $J$ express article 10.1096/fj.04-2695fje. Published online October 26, 2004.

33. Biben C, Harvey RP. Homeodomain factor Nkx-2.5 controls left/right asymmetric expression of bHLH gene eHand during heart development. Genes Dev 1997;11:1357-69.

34. Lints TJ, Parsons LM, Hartley L, Lyons I, Harvey RP. Nkx-2.5: a novel murine homeobox gene expressed in early heart progenitor cells and their myogenic descendants. Development 1993;119:419-31.

35. Benson DW, Silberbach GM, Kavanaugh-McHugh A, Cottrill C, Zhang Y, Riggs S, et al. Mutations in the cardiac transcription factor Nkx-2.5 affect diverse cardiac developmental pathways. J Clin Invest 1999;104:1567-73.

36. Sengupta S, Toh SA, Sellers LA, Skepper JN, Koolwijk P, Leung HW, et al. Modulating angiogenesis: the yin and the yang in ginseng. Circulation 2004;110:1219-25.
37. Shen LH, Zhang JT. Ginsenoside Rg1 promotes proliferation of hippocampal progenitor cells. Neurol Res 2004;26:422-8.

38. Lee YN, Lee HY, Chung HY, Kim SI, Lee SK, Park BC, et al. In vitro induction of differentiation by ginsenoides in F9 teratocarcinoma cells. Eur J Cancer 1996;32A(8):1420-8.

39. Aicher A, Heeschen C, Mildner-Rihm C, Urbich C, Ihling C, Technau-Ihling K, et al. Essential role of endothelial nitric oxide synthase for mobilization of stem and progenitor cells. Nat Med 2003;9:1370-6.

40. Bloch W, Fleischmann BK, Lorke DE, Andressen C, Hops B, Hescheler J, et al. Nitric oxide synthase expression and role during cardiomyogenesis. Cardiovasc Res 1999;43:675-84.

41. Mangi AA, Noiseux N, Kong D, He H, Rezvani M, Ingwall JS, et al. Mesenchymal stem cells modified with Akt prevent remodeling and restore performance of infarcted hearts. Nat Med 2003;9: 1195-201.

42. Pittenger MF, Martin B.J. Mesenchymal stem cells and their potential as cardiac therapeutics. Circ Res 2004;95:9-20.

43. Velculescu VE, Zhang L, Vogelstein B, Kinzler KW. Serial analysis of gene expression. Science 1995;270:484-7.

44. Schena M, Shalon D, Davis RW, Brown PO. Quantitative monitoring of gene expression patterns with a complementary DNA microarray. Science 1995;270:467-70

Received April 28, 2005; accepted June 22, 2005 


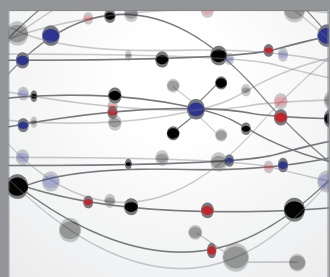

The Scientific World Journal
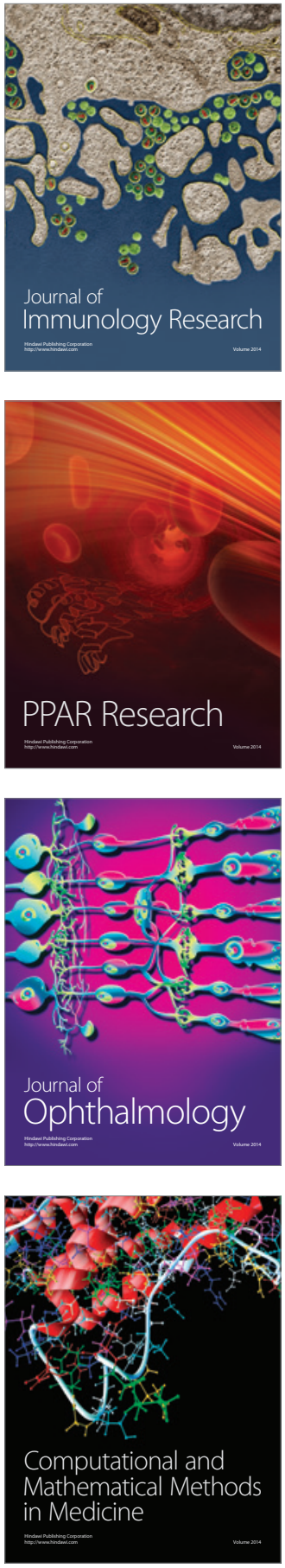

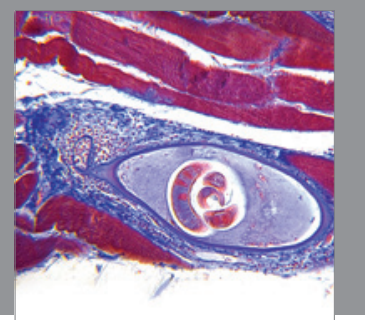

Gastroenterology

Research and Practice
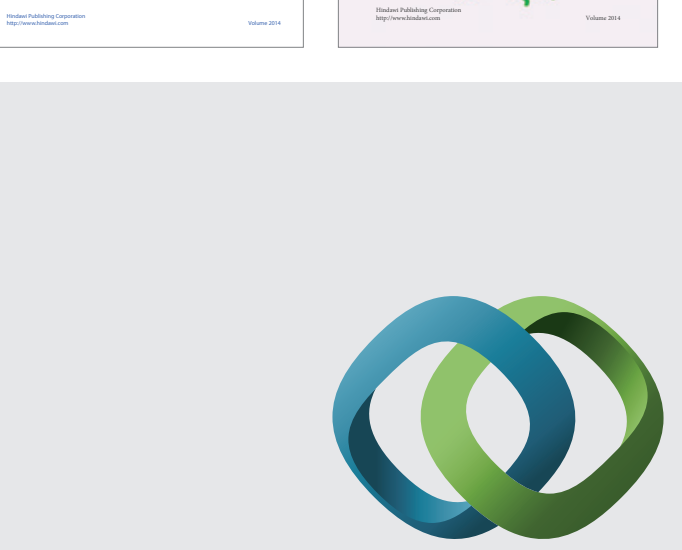

\section{Hindawi}

Submit your manuscripts at

http://www.hindawi.com
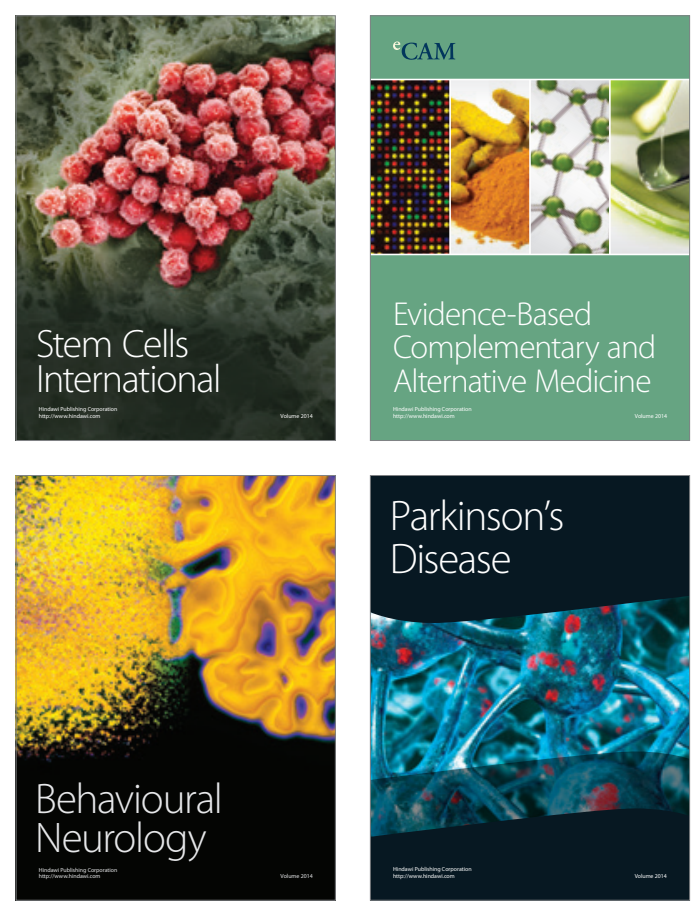

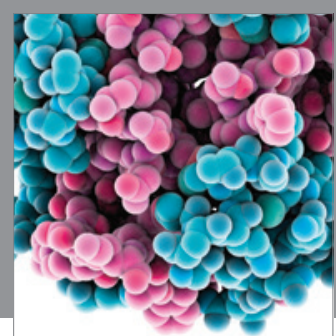

Journal of
Diabetes Research

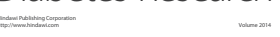

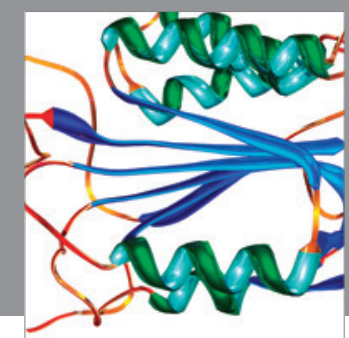

Disease Markers
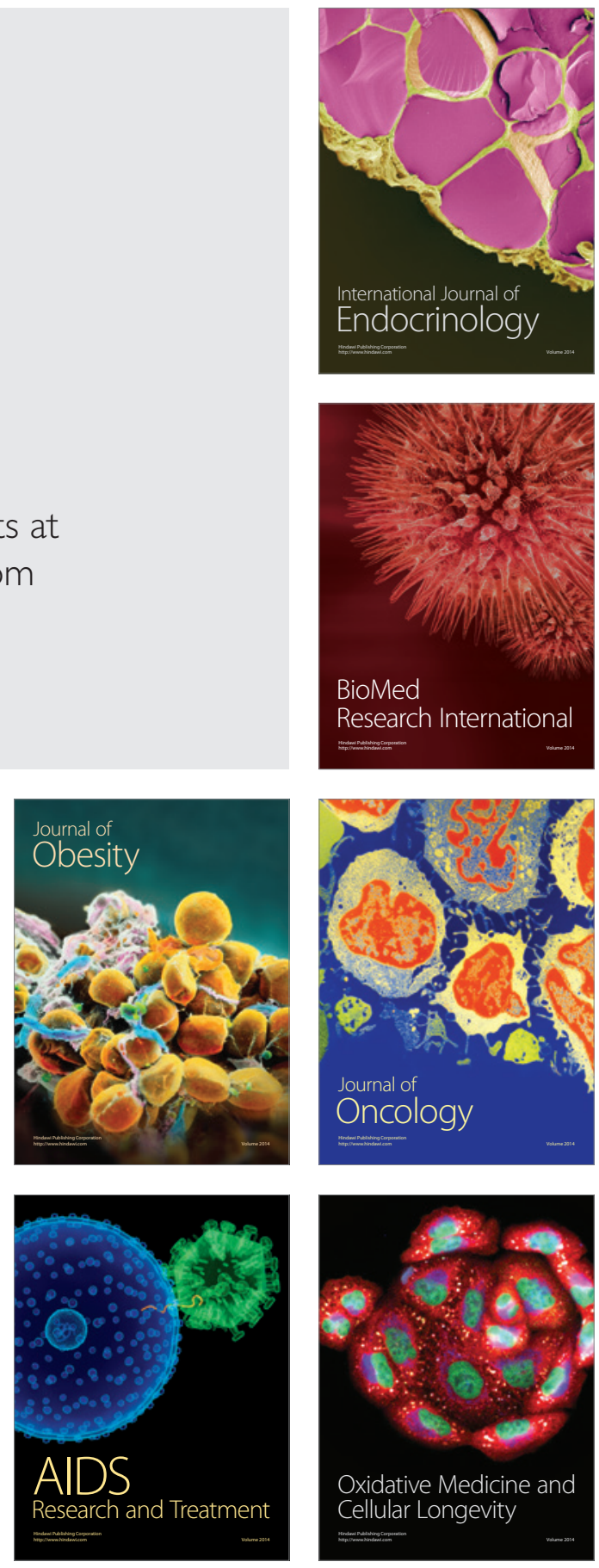